\title{
EDITORIAL
}

\section{Biomarkers in Alzheimer disease: are we there yet?}

\author{
Jaqueline S. Generoso, ${ }^{1}$ iD Rodrigo Morales, ${ }^{2,3}$ Tatiana Barichello ${ }^{1,4}$ iD \\ ${ }^{1}$ Laboratório de Fisiopatologia Experimental, Programa de Pós-Graduação em Ciências da Saúde, Universidade do Extremo Sul Catarinense \\ (UNESC), Criciúma, SC, Brazil. ${ }^{2}$ Department of Neurology, McGovern Medical School, The University of Texas Health Science Center at \\ Houston, Houston, TX, USA. ${ }^{3}$ Centro Integrativo de Biología y Química Aplicada (CIBQA), Universidad Bernardo O'Higgins, Santiago, Chile. \\ ${ }^{4}$ Translational Psychiatry Program, Department of Psychiatry and Behavioral Sciences, McGovern Medical School, The University of Texas \\ Health Science Center at Houston, Houston, TX, USA.
}

The search for meaningful biomarkers is one of the hottest areas of Alzheimer research. These biomarkers should be specific, suitable for routine use in clinical practice, and indicate specific stages of the disease. Nevertheless, Alzheimer disease diagnosis is mostly based on clinical findings. Several potential Alzheimer disease biomarkers have been evaluated in blood and cerebrospinal fluid (CSF) and through brain imaging techniques (Figure 1). Recently, Pais et al. discussed methods that are either established or under development for early Alzheimer disease diagnosis. ${ }^{1}$

The most-cited blood and CSF biomarkers for diagnosing Alzheimer disease include the 42-amino acid form of amyloid-beta peptide $\left(A \beta_{1-42}\right)$, the $A \beta_{1-42} / A \beta_{1-40}$ ratio, total and phosphorylated fractions of tau proteins (T-Tau and $\mathrm{P}-\mathrm{Tau}$, respectively), neurofilament light chain (NFL) protein, phosphorylated tau at position threonine 181 ( $\mathrm{P}$-Tau 181), and 217 (P-Tau217). It has been reported that in Alzheimer patients the $A \beta_{1-42} / A \beta_{1-40}$ ratio decreases 14$20 \%$ in plasma and $50 \%$ in CSF, although plasma levels of T-Tau, P-Tau, NFL, and P-Tau181 increase. Significantly, NFL levels were found to be higher in Alzheimer patients and persons with mild cognitive impairment than in healthy controls. A longitudinal increase in NFL plasma levels also correlates with low CSF $A \beta_{1-42}$ levels, high CSF levels of T-Tau and P-Tau, along with low brain fluorodeoxyglucose-positron emission tomography (PET) uptake. ${ }^{2}$ Recent publications have demonstrated that plasma PTau181 levels can predict long-term cognitive decline in healthy older adults. P-Tau181 was found to be 3.5 times higher in the plasma of Alzheimer patients than controls and frontotemporal dementia patients. Plasma P-Tau181 levels have been associated with CSF levels of PTau181, as well as with A $\beta$-PET and cortical tau protein deposition when measured by $\left[{ }^{18} \mathrm{~F}\right]$ flortaucipir PET. ${ }^{3}$ These results demonstrate that plasma P-Tau181 could be a promising Alzheimer disease biomarker.

CSF biomarkers have an advantage over peripheral blood markers in their direct contact with the brain and spinal cord. CSF, which represents various biochemical

Correspondence: Tatiana Barichello, Department of Psychiatry and Behavioral Sciences, McGovern Medical School, The University of Texas Health Science Center at Houston, 1941 East Road, Suite 3140, Houston, TX, 77054, USA.

E-mail: Tatiana.Barichello@uth.tmc.edu

Submitted May 23 2020, accepted May 23 2020, Epub Jul 102020. and metabolic profiles in the brain, could be a valuable source of information. The established CSF biomarkers for Alzheimer disease diagnosis include low levels of $A \beta_{1-42}$ and the $A \beta_{1-42} / A \beta_{1-40}$ ratio, increased levels of T-Tau, P-Tau, NFL, P-Tau181, P-Tau217, and growth-associated protein 43 (GAP-43). ${ }^{4}$ Alzheimer disease triggers an accumulation of $A \beta_{1-42}$ in the brain and, as a consequence, $A \beta_{1-42}$ levels decrease in the CSF and bloodstream. CSF levels of T-tau and P-tau are elevated due to cortical neuronal loss and cortical tangle formation, respectively, while increased NFL levels are associated with neuroaxonal damage. P-Tau181 and P-Tau217 reflect abnormalities of tau metabolism in the brain. CSF levels of P-Tau181 are higher in Alzheimer patients than in other tauopathies, such as frontotemporal dementia. Importantly, the association between CSF P-Tau181 and Tau PET measures is moderate in Alzheimer disease and low in other dementias. ${ }^{5}\left[{ }^{18} \mathrm{~F}\right]$ flortaucipir and $\left[{ }^{18} \mathrm{~F}\right] \mathrm{flu}$ temetamol uptake, as well as CSF $A \beta_{1-42}$, were more highly correlated with CSF levels of P-Tau217 than CSF P-Tau181. ${ }^{5}$ This indicates that P-Tau could be a compelling marker for Alzheimer disease diagnosis. High levels of GAP-43, a membrane protein related to the cytoplasmic area of axonal growth cones, were recently identified in the CSF of Alzheimer patients and were associated with cognitive impairment. The specificity of CSF levels of GAP-43 is supported by their association with neurofibrillary tangles and $A \beta$ plaques in the hippocampus, amygdala, and cortex. These findings were not observed for $\alpha$-synuclein or TAR DNA-binding protein 43 (TDP-43) pathology. ${ }^{6}$

Tau-selective PET tracers facilitate investigation of tau pathology in neurodegenerative disorders. Although $\left[{ }^{18} \mathrm{~F}\right]$ flortaucipir differentiates Alzheimer disease from other neurodegenerative disorders, this tracer also shows high retention in the brains of the patients with a semantic variant of primary progressive aphasia. Other tauselective PET tracers, including $\left[{ }^{18} \mathrm{~F}\right] \mathrm{GTP} 1$ (Genentech tau probe 1), $\left[{ }^{18} \mathrm{~F}\right] \mathrm{MK}-6240$, and $\left[{ }^{18} \mathrm{~F}\right] \mathrm{RO} 048$, could overcome the potential confounding issues mentioned above.

How to cite this article: Generoso JS, Morales R, Barichello T. Biomarkers in Alzheimer disease: are we there yet? Braz J Psychiatry. 2020;42:337-339. http://dx.doi.org/10.1590/1516-44462020-0013 


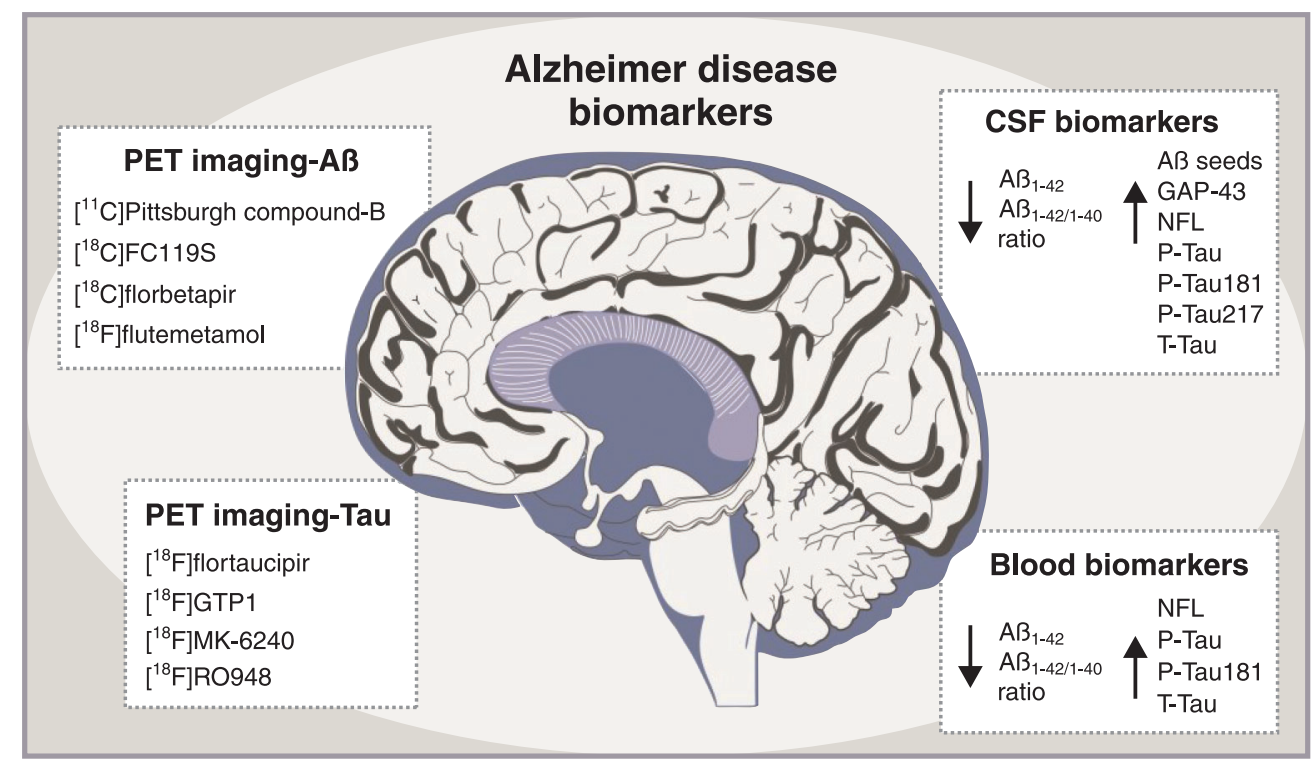

Figure 1 Biomarkers used in Alzheimer disease diagnosis. In Alzheimer disease, levels of the 42-amino acid form of $A \beta_{1-42}$ and the $A \beta_{1-42 / 1-40}$ ratio are low in plasma and CSF. Plasma and CSF levels of T-Tau, P-Tau, P-Tau181, and NFL protein are high. CSF levels of GAP-43 and P-Tau217 are also high. A $\beta$ PET selective tracers include: Pittsburgh compound-B [ $\left.{ }^{11} \mathrm{C}\right]-\mathrm{PIB}$, $\left[{ }^{18} \mathrm{~F}\right]$ florbetapir, $\left[{ }^{18} \mathrm{~F}\right]$ flutemetamol, and $\left[{ }^{18} \mathrm{~F}\right] \mathrm{FC} 119 \mathrm{~S}$. Tau-selective PET tracers include: $\left[{ }^{18} \mathrm{~F}\right] \mathrm{flortaucipir},\left[{ }^{18} \mathrm{~F}\right] \mathrm{GTP} 1,\left[{ }^{18} \mathrm{~F}\right] \mathrm{MK}$ 6240, and [ $\left.{ }^{18} \mathrm{~F}\right] \mathrm{RO}$ 948. A $\beta$ = amyloid-beta; CSF = cerebrospinal fluid; GAP-43 = growth-associated protein 43; GTP1 = Genentech tau probe 1; NFL = neurofilament light chain; PET = positron emission tomography; PIB = Pittsburgh compound-B; $\mathrm{P}-\mathrm{Tau}=$ phosphorylated tau protein; $\mathrm{P}-\mathrm{Tau} 181=$ phosphorylated tau at position threonine $181 ; \mathrm{P}-\mathrm{Tau} 217=$ tau phosphorylated at position threonine 217; T-Tau = total tau protein.

For example, $\left[{ }^{18} \mathrm{~F}\right] \mathrm{R} 0948$ had a high specificity for Alzheimer disease-type paired helical tau filaments outside the medial temporal lobe. ${ }^{7}$

Amyloid- $\beta$ PET tracers, including the Pittsburgh compound-B $\left[{ }^{11} \mathrm{C}\right]-\mathrm{PIB}, \quad\left[{ }^{18} \mathrm{~F}\right]$ florbetapir, $\left[{ }^{18} \mathrm{~F}\right]$ flutemetamol, and $\left[{ }^{18} \mathrm{~F}\right] \mathrm{FC} 119 \mathrm{~S}$, can be used for longitudinal tracking of Alzheimer progression. Evaluating biomarkers through PET imaging in combination with CSF biochemical profiles may be essential for identifying brain areas where $A \beta$ accumulation begins in patients, including increased levels of CSF $A \beta_{1-42}$ but a negative $A \beta$ PET scan. ${ }^{8}$

The emergence of novel biomarkers, such specific microRNA populations, downstream molecules associated with $A \beta$ and tau toxicity, and ultrasensitive detection of seeding-competent $A \beta$ and tau populations are currently being explored for use as early, low-invasive, sensitive, and specific diagnostic probes. Forthcoming research will reveal whether the coveted diagnostic test for Alzheimer disease is a real possibility.

In conclusion, diagnostic tests that identify individuals for early treatment will have great significance and value. A blood-based Alzheimer test (i.e. low cost and minimally-invasive) would be preferable to the currently approved CSF and PET diagnostic tests. Plasma $A \beta_{1-42}$, the $A \beta_{1-42 / 1-40}$ ratio, and $\mathrm{P}-\mathrm{Tau181}$ may be beneficial for amyloid and tau pathology screening, while NFL may be useful for monitoring the progression of Alzheimer-type dementia. Currently under development, seeding aggregation assays ${ }^{9}$ are extremely sensitive techniques that exploit the functional properties of $A \beta$ oligomers to seed the polymerization of monomeric $A \beta$. Recent advances in such innovative techniques may result in a blood-based diagnostic test for Alzheimer disease.

\section{Acknowledgments}

The authors would like to thank The University of Texas Health Science Center at Houston (UTHealth), the Universidade do Extremo Sul Catarinense (UNESC), the Conselho Nacional de Desenvolvimento Científico e Tecnológico (CNPq), and the Fundação de Amparo à Pesquisa e Inovação de Santa Catarina (FAPESC). TB has received grants from the Alzheimer's Association ${ }^{\circledR}$ (AARGDNTF-19-619645).

\section{Disclosure}

The authors report no conflicts of interest.

\section{References}

1 Pais M, Martinez L, Ribeiro O, Loureiro J, Fernandez R, Valiengo L, et al. Early diagnosis and treatment of Alzheimer's disease: new definitions and challenges. Braz J Psychiatry. 2020;42:431-41.

2 Mattsson N, Cullen NC, Andreasson U, Zetterberg H, Blennow K. Association between longitudinal plasma neurofilament light and neurodegeneration in patients with Alzheimer disease. JAMA Neurol. 2019;76:791-9.

3 Thijssen EH, La Joie R, Wolf A, Strom A, Wang P, laccarino L, et al. Diagnostic value of plasma phosphorylated tau181 in Alzheimer's disease and frontotemporal lobar degeneration. Nat Med. 2020; 26:387-97.

4 Barichello T, Giridharan VV, Dal-Pizzol F. A cerebrospinal fluid biosignature for the diagnosis of Alzheimer's disease. Braz J Psychiatry. 2019;41:467-8. 
5 Janelidze S, Stomrud E, Smith R, Palmqvist S, Mattsson N, Airey DC, et al. Cerebrospinal fluid p-tau217 performs better than p-tau181 as a biomarker of Alzheimer's disease. Nat Commun. 2020;11:1683.

6 Sandelius A, Portelius E, Källén A, Zetterberg H, Rot U, Olsson B, et al. Elevated CSF GAP-43 is Alzheimer's disease specific and associated with tau and amyloid pathology. Alzheimers Dement. 2019;15:55-64.

7 Leuzy A, Smith R, Ossenkoppele R, Santillo A, Borroni E, Klein G, et al. Diagnostic performance of RO948 F 18 tau positron emission tomography in the differentiation of alzheimer disease from other neurodegenerative disorders. JAMA Neurol. 2020 May 11;e200989. doi: 10.1001/jamaneurol.2020.0989. Online ahead of print.

8 Mattsson N, Palmqvist S, Stomrud E, Vogel J, Hansson O. Staging $\beta$-amyloid pathology with amyloid positron emission tomography. JAMA Neurol. 2019 Jul 17;76(11). doi: 10.1001/jamaneurol.2019. 2214. Online ahead of print.

9 Salvadores N, Shahnawaz M, Scarpini E, Tagliavini F, Soto C. Detection of misfolded $A \beta$ oligomers for sensitive biochemical diagnosis of Alzheimer's disease. Cell Rep. 2014;7:261-8. 